\title{
On the Impulse Method for Cloth Animation
}

\author{
Juntao Ye, Robert E. Webber, and Irene Gargantini \\ The University of Western Ontario, London, Ontario, N6A 5B7 Canada \\ \{juntao, webber, irene\}@csd.uwo.ca
}

\begin{abstract}
Computer animation of cloth is often plagued by springs being overstretched. Our approach addresses this problem and presents some preliminary results.
\end{abstract}

\section{Introduction}

Cloth animation faces two major challenges: realism and speed. The way these two issues are addressed and resolved largely depends on the model adopted. So far the most successful system for creating realistic folds and creases as cloth is subject to various forces (gravity, for instance) has been the mass-spring model. Here the fabric is represented as a 2D array of nodes, each with a given mass, and each being related to its neighbors by mutual stretching, shearing and bending. Overstretching, however, appears in all the work presented in the literature. It is counteracted, but not completely eliminated, in different ways, such as adjusting nodes position Pro95, adjusting nodes velocity VSC01, using momentum transfer [VCM95, and applying impulses to the nodes [BFA02. We present a new method based on the linearization of a nonlinear formulation of impulse calculation. Applying this new impulse approach to cloth animation solves satisfactorily both overstretching and overcompression. Although not shown here, it turns out that it can be proven [Ye05] that the matrix of the linearized system is symmetric, positive definite. This allows more efficient solvers to be used thus decreasing the computational burden always present in cloth animation, especially in the instances of collision detection and resolution.

\section{The Mass-Spring Model}

Our cloth model consists of three kinds of springs: stretching springs to model the response of the cloth when pulled in the direction of its threads; shearing springs to simulate the response of the cloth when pulled diagonally; bending springs to model cloth resistance to out-of-plane forces (see Figure1 and 2). The stretching spring is linear, while both shearing and bending are angular. The bending spring (not shown) is mounted on the common edge of every pair of adjacent triangles, its movement taking place in a plane normal to both triangles. Note that our bending model is borrowed from Bridson et al. BMF03, and that the shear force $\mathbf{f}^{h}$ exerted on three nodes in Figure 1 is defined as

$$
\mathbf{f}_{i}^{h}=k^{h} \cos \theta \mathbf{u}_{i}, \text { for } i=1,2,3
$$




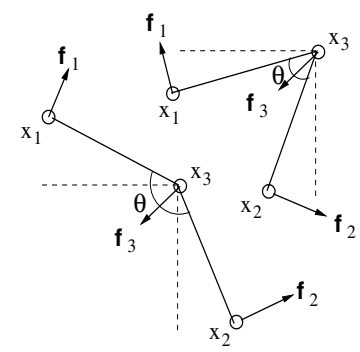

Fig. 1. The shear force model with rest angle $\theta_{0}=\pi / 2$

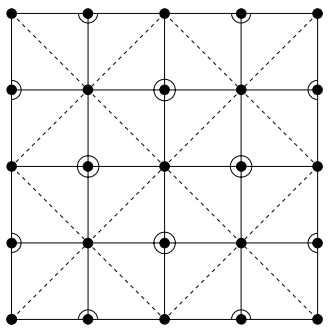

Fig. 2. An isotropic cloth mesh, each right-angle arc represents a shear spring

where $k^{h}$ is the shear coefficient and

$$
\begin{gathered}
\mathbf{u}_{1}=\frac{1}{\left|\mathbf{r}_{1}\right|} \frac{\mathbf{r}_{1} \times\left(\mathbf{r}_{1} \times \mathbf{r}_{2}\right)}{\left|\mathbf{r}_{1} \times\left(\mathbf{r}_{1} \times \mathbf{r}_{2}\right)\right|}, \quad \mathbf{u}_{2}=\frac{1}{\left|\mathbf{r}_{2}\right|} \frac{\left(\mathbf{r}_{1} \times \mathbf{r}_{2}\right) \times \mathbf{r}_{2}}{\left|\left(\mathbf{r}_{1} \times \mathbf{r}_{2}\right) \times \mathbf{r}_{2}\right|}, \\
\mathbf{u}_{3}=-\mathbf{u}_{1}-\mathbf{u}_{2}, \quad \mathbf{r}_{1}=\mathbf{x}_{1}-\mathbf{x}_{3}, \quad \mathbf{r}_{2}=\mathbf{x}_{2}-\mathbf{x}_{3} .
\end{gathered}
$$

Thus one angular spring generates three forces (one for each node in the angle). If $k^{d}$ is the damping coefficient, the shear damping $\mathbf{f}^{d}$ for these nodes is

$$
\mathbf{f}_{i}^{d}=-k^{d} \frac{d \theta}{d t} \mathbf{u}_{i}, \quad \text { where } \frac{d \theta}{d t}=\mathbf{u}_{1} \cdot \mathbf{v}_{1}+\mathbf{u}_{2} \cdot \mathbf{v}_{2}+\mathbf{u}_{3} \cdot \mathbf{v}_{3} .
$$

Thus the shearing model is a 2D version of the bending model BMF03.

\section{Constructing the Impulse Equation System}

Since $\mathbf{F} \delta t=m \delta \mathbf{v}$, using impulses instead of forces offers an advantage. Whenever forces are used, in fact, we need to know their magnitude and the time during which they are in effect. Impulse, on the other hand, is directly related to velocity change. The strain limits for the stretching and compression, $L^{\mathrm{c}}$ and $L^{\mathrm{s}}$, are set ahead of time by the user. A spring is neither allowed to stretch more than $L^{\mathrm{s}}$, nor allowed to shrink less than $L^{\mathrm{c}}$. Whenever $L \in\left[L^{\mathrm{c}}, L^{\mathrm{s}}\right]$, the spring tension is a linear function of the spring length according to $\mathbf{f}=k\left(\left|\mathbf{x}_{j}-\mathbf{x}_{i}\right|-l_{i j}^{0}\right) \frac{\mathbf{x}_{j}-\mathbf{x}_{i}}{\left|\mathbf{x}_{j}-\mathbf{x}_{i}\right|}$, where $l_{i j}^{0}$ is the rest length of the spring connecting $x_{i}$ and $x_{j}$. When a spring is, or potentially will be, out of the limits, impulses are generated and applied to the two end-nodes so that the spring length after the next time step is within the given range. This method works as if there were a massless string and a massless rod passing through the spring (see Figure 3). This string with length $L^{\mathrm{s}}$ is non-stretchable and this rod with length $L^{\mathrm{c}}$ is non-compressible.

A spring $x_{i j}$, connecting node $x_{i}$ and $x_{j}$ with $i<j$, generates impulses $I_{i j}$ to $x_{i}$ and $-I_{i j}$ to $x_{j}$, their directions being collinear with that of $\mathbf{x}_{i j}$. This way, the impulses are considered to be created exclusively by internal forces. When there is no external force acting on a system, both the linear momentum and the 
angular momentum of the system are conserved. We define $I_{i j}=s_{i j} \hat{\mathbf{x}}_{i j}$, where $\hat{\mathbf{x}}_{i j}=\frac{\mathbf{x}_{i j}}{\left|\mathbf{x}_{i j}\right|}$ and $s_{i j}$ is the magnitude of the impulse which we want to compute. Thus node $\mathbf{x}_{i}$ receives velocity change $\frac{s_{i j} \hat{\mathbf{x}}_{i j}}{m_{i}}$ and node $\mathbf{x}_{j}$ receives velocity change $-\frac{s_{i j} \hat{\mathbf{x}}_{i j}}{m_{j}}$, the positive sign being attributed to the node having smaller index. A node receives impulses from all its incident springs. Consider now seven springs connecting eight nodes, with the node indices satisfying $f<g<h<i<j<$ $k<l<n$ (see Figure 4). The velocity changes for nodes $x_{i}$ and $x_{j}$ are

$$
\begin{gathered}
\delta \mathbf{v}_{i}=\left(s_{i j} \hat{\mathbf{x}}_{i j}+s_{i l} \hat{\mathbf{x}}_{i l}-s_{f i} \hat{\mathbf{x}}_{f i}-s_{h i} \hat{\mathbf{x}}_{h i}\right) / m_{i}, \\
\delta \mathbf{v}_{j}=\left(-s_{i j} \hat{\mathbf{x}}_{i j}-s_{g j} \hat{\mathbf{x}}_{g j}+s_{j k} \hat{\mathbf{x}}_{j k}+s_{j n} \hat{\mathbf{x}}_{j n}\right) / m_{j} .
\end{gathered}
$$

Suppose at time $t_{0}$, the spring length is $L^{t_{0}}=\left|\mathbf{x}_{j}-\mathbf{x}_{i}\right|$. Once the ODE solver computes the new velocities $\mathbf{v}_{i}$ and $\mathbf{v}_{j}$, the nodes will move to new positions accordingly. We can predict the spring length at time $t_{0}+h$ to be $\tilde{L}^{t_{0}+h}=$ $\left|\left(\mathbf{x}_{j}+\mathbf{v}_{j} h\right)-\left(\mathbf{x}_{i}+\mathbf{v}_{i} h\right)\right|$. If $\tilde{L}^{t_{0}+h} \notin\left[L^{\mathrm{c}}, L^{\mathrm{s}}\right]$, the spring will be overstretched or overcompressed and we use impulses to change the node velocities so that the new spring length

$$
L^{t_{0}+h}=\left|\mathbf{x}_{j}-\mathbf{x}_{i}+\left(\mathbf{v}_{j}+\delta \mathbf{v}_{j}\right) h-\left(\mathbf{v}_{i}+\delta \mathbf{v}_{i}\right) h\right|
$$

satisfies $L^{t_{0}+h} \in\left[L^{\mathrm{c}}, L^{\mathrm{s}}\right]$. We can choose the value for $L^{t_{0}+h}$ according to the value of $\tilde{L}^{t_{0}+h}$ :

$$
L^{t_{0}+h}= \begin{cases}L^{\mathrm{s}} & \text { if } \tilde{L}^{t_{0}+h}>L^{\mathrm{s}} \\ L^{\mathrm{c}} & \text { if } \tilde{L}^{t_{0}+h}<L^{\mathrm{c}} \\ \tilde{L}^{t_{0}+h} & \text { otherwise }\end{cases}
$$

Since each stretching spring corresponds to one equation like Equ. 3, we get a system of nonlinear equations. Using Equ. 1 and 2, Equ. 3 becomes a function of $s_{i j}$ terms. If an appropriate method can solve this nonlinear system of equations, then it is guaranteed that none of the springs will be over-stretched or overcompressed. Methods for solving such nonlinear systems tend to be very slow so a linearization is in order. This approximation is not guaranteed to result in every spring being within the limits after the impulse application, but in our experiments, it always produced springs that are within the limits. Details of the linearization can be found in [Ye05].

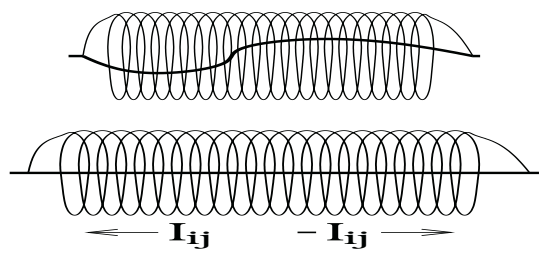

Fig. 3. Spring with string and rod

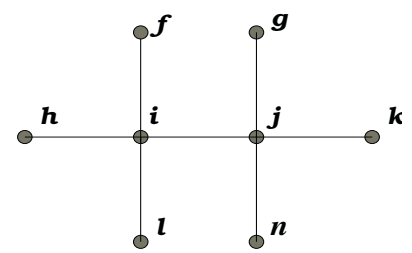

Fig. 4. Seven neighboring springs 


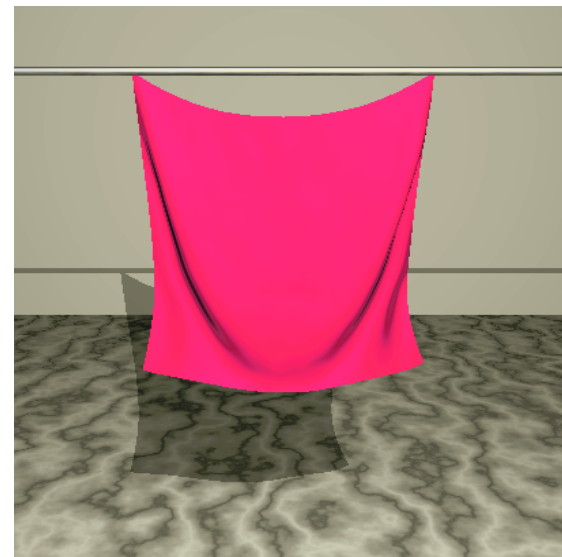

Fig. 5. Cloth suspended from two corners

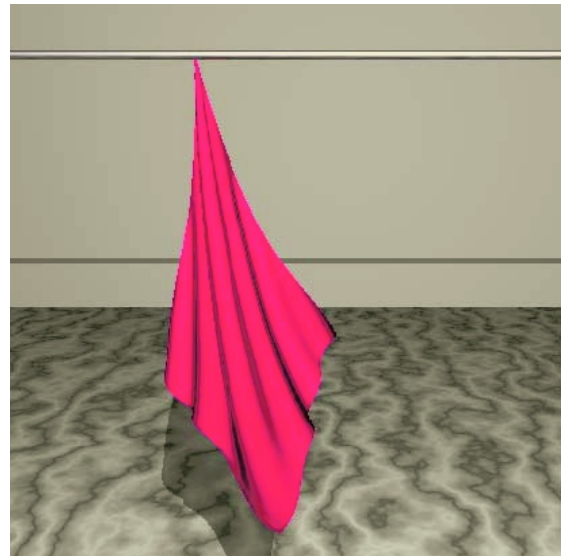

Fig. 6. Cloth swinging down

\section{$4 \quad$ Results and Conclusion}

Figure 5 shows the cloth suspended from two corners held $75.2 \mathrm{~cm}$ apart. Excessive stretching in mass-spring cloth models would typically appear near the top corners of the cloth. Notice that the cloth around these corners in this figure does not appear overstretched. Figure [6] shows the cloth 1.6 seconds after releasing the top-right corner, while it is still swinging. With the cloth suspended from only one corner, even more force is being applied to the springs at the top-left corner, but they are still not overstretched.

\section{References}

[BFA02] Robert Bridson, Ronald Fedkiw, and John Anderson. Robust treatment of collisions, contact and friction for cloth animation. ACM Transactions on Graphics (SIGGRAPH '02), 21(3):594-603, 2002.

[BMF03] Robert Bridson, S. Marino, and Ronald Fedkiw. Simulation of clothing with folds and wrinkles. In Proceedings of SIGGRAPH/Eurographics Symposium on Computer Animation (SCA 2003), pages 28-36, 2003.

[Pro95] Xavier Provot. Deformation constraints in a mass-spring model to describe rigid cloth behavior. In Graphics Interface '95, pages 147-154, 1995.

[VCM95] Pascal Volino, Martin Courchesne, and Nadia Magnenat Thalmann. Versatile and efficient techniques for simulating cloth and other deformable objects. In Proceedings of SIGGRAPH '95, pages 137-144, 1995.

[VSC01] T. Vassilev, B. Spanlang, and Y. Chrysanthou. Fast cloth animation on walking avatars. In EUROGRAPHICS '01, volume 20, pages 260-267, 2001.

[Ye05] Juntao Ye. Computational Aspects of the Dynamics of Cloth. PhD thesis, Department of Computer Science, The University of Western Ontario, in progress 2005. 